\title{
Rubella immunity among pregnant women in Iran
}

\begin{abstract}
Background: Although rubella is a benign dermatose in childhood, when infection develops during pregnancy, especially during the first 12 weeks, it often causes congenital rubella syndrome (CRS) resulting in abortion and severe congenital defects. Thus, the major goal of the rubella vaccination program is the prevention of congenital rubella syndrome. This study was performed to assess the seroprevalence of rubella virus infection among pregnant women in Birjand, Iran.
\end{abstract}

Methods: In this cross-sectional study, one hundred serum specimens from 100 pregnant women were randomly selected in Birjand, Iran. The specimens were tested for rubella immunoglobulin $\mathrm{G}$ antibodies using a commercial immunoassay.

Results: A total of $94 \%$ of the pregnant women were immune to rubella. According to age group, a total of $88.2 \%$ of women in the 15 - 19-year age group, $94.7 \%$ in the $20-24-$ year age group, $93.8 \%$ in the 25 - 29-year age group, $100 \%$ of women in the $30-34$-year age group and $100 \%$ in the 35-39-year age group were immune to rubella. There was no statistically significant difference in the rate of rubella susceptibility between the groups tested.

Conclusion: Although the present study showed that the level of immunity among women in Birjand is greater than the predicated critical level, but it is recommended that all the women be checked serologically for $\mathrm{IgG}$ against rubella virus prior to marriage and seronegative women be vaccinated against rubella.

Keywords: immunity, Iran, pregnant women, rubella
Volume 4 Issue 2 - 2016

Faezeh Ghaderi, ${ }^{3}$ Reza Ghaderi',2

'Department of Dermatology, Birjand University of Medical Sciences, Iran

2Medical Toxicology Research Center, Mashhad University of

Medical Sciences, Iran

Correspondence: Faezeh Ghaderi, Associate professor, Pediatric Dentistry Department, Shiraz University of Medical, Science, Shiraz, Iran \& Oral and Dental Disease Research Center, School of Dentistry, Shiraz University of Medical Science, Shiraz, Iran, Email faezeghaderi@yahoo.com

Received:September 13, 2016 | Published: October 06, 2016

\section{Introduction}

Rubella was first known as a type of measles but later on was called Rotheln or German measles, and after the year 1866 was given the name Rubella. Rubella is a common dermatose in children. Its importance is due to the teratogenic effects of rubella infection in the first 12 weeks of pregnancy. ${ }^{1,2}$

Rubella infection developed in the first trimester of pregnancy is associated with about $90 \%$ risk of congenital rubella syndrome. Thus, the major goal of the rubella vaccination program is the prevention of congenital malformation. ${ }^{2}$

Therefore, vaccination is the most important way to prevent rubella and, consequently congenital malformation. ${ }^{1}$

A very efficient vaccine has been available since 1969 and vaccination programs have greatly reduced the incidence of rubella in developed countries. ${ }^{2,3}$

Information on the epidemiology of rubella in Iran is still insufficient and the frequency of non immune women of childbearing age is unknown.

In other developing regions, the percentage of women of childbearing age susceptible to rubella varies from $4 \%$ in China to $70 \%$ in Trinidad and Tobago. ${ }^{2,4}$

This study was conducted to evaluate the seroprevalence of rubella virus infection among pregnant women aged between 15 and 39 years in Birjand, Iran.

\section{Materials and methods}

In this prospective cross-sectional study, 100 pregnant women were randomly selected. These subjects comprised 100 pregnant women who presented at the Department of Gynecology of Birjand
University of Medical Sciences, Birjand, in the center of south Khorasan province in the east of Iran.

They were randomly enrolled after obtaining verbal consent to participate in the study. Approval of the Institutional Ethical Committee was obtained, Demographic data such as age and other information including resident place (urban, rural), occupation and history of vaccination were collected using a checklist. Patients with positive history of measles, mumps, and rubella) MMR (vaccination were excluded from the study. One hundred serum specimens from 100 pregnant women were selected. The specimens were age stratified and screened for rubella immunoglobulin G (IgG) antibodies by means of a commercial immunoassay (Elecsys rubella IgG, Roche Diagnostics, Mannheim, Germany).

The collected data were analyzed using SPSS (version 22) software, $\mathrm{T}$ and chi-square tests. Test of significance was carried out at $5 \%$ level of significance.

\section{Results}

The mean $( \pm \mathrm{SD})$ age of the pregnant women was 22.9 ( \pm 3.9$)$. A total of 100 serum specimens were tested and included in the analysis. The numbers and rate of immune pregnant woman in different groups was shown in Table 1.

Using the chi-square test no statistically significant difference in the rate of rubella susceptibility was found between the groups tested $(\mathrm{p}=0.08)$. The mean age of women with positive $(23.1 \pm$ $3.9)$ and negative rubella serology $(20.6 \pm 3.1)$ was not significantly different. The percentage of immune women was \%94.6 and $93.9 \%$ in households and employers, respectively. There was no significant difference between immune households and employers $(\mathrm{p}=0.09)$.

The percentage of immune women was 95.5 and 92.5 in rural and urban women, respectively. There was no significant difference between immune rural and urban women $(\mathrm{p}=0.20)$. 
Table I Numbers and rate of immune pregnant women to rubella in different age groups

\begin{tabular}{llll}
\hline Age Group & $\begin{array}{l}\text { Numbers } \\
\text { in each } \\
\text { Group }\end{array}$ & $\begin{array}{l}\text { Numbers } \\
\text { who } \\
\text { Immune to } \\
\text { Rubella }\end{array}$ & $\begin{array}{l}\text { Rate of } \\
\text { Immune } \\
\text { to Rubella }\end{array}$ \\
\hline I5-19 Years old & 17 & 15 & $88.20 \%$ \\
20-24 Years old & 19 & 18 & $94.70 \%$ \\
25-29 Years old & 16 & 15 & $93.80 \%$ \\
30-34 Years old & 25 & 25 & $100 \%$ \\
35-39 Years old & 23 & 23 & $100 \%$ \\
\hline
\end{tabular}

P-value $>0.05$

\section{Discussion}

Knowledge of age-specific rubella immunity, especially the rate of women of childbearing age who are susceptible to rubella, is an essential element to manage an efficient rubella vaccination program. A very efficient vaccine has been available since 1969 and vaccination programs have greatly reduced the incidence of rubella and congenital malformations in developed countries.,3

Information on the epidemiology of rubella in Iran is still incomplete. An article entitled 'Role of rubella in patients with acute febrile rash in different parts of Iran'as reported 'Acute rubella infection in $51.8 \%$ of all 1169 measles negative cases. This rate was 63.6, 61 and 29.7 percent for the north, central and southern part of Iran, respectively. Relative frequency of acute rubella in patients with skin rash under 15 years-old was $31.1 \%$ compared to $7.4 \%$ in those equal and older than 15 years-old. ${ }^{5}$

In another study 6, a community-based rubella serological survey of 770 individuals selected by cluster sampling from rural Urmia, the center of west Azerbaijan province located in northwestern Iran, was carried out from December 2001 to March 2002. The aim of the study was to determine rubella epidemiology in a representative non-immunized community in northwestern Iran and also to assess national vaccination program against rubella. The study comprised a seroprevalence study, with stratification by sex and age (0-45 years). Sera from 392 females and 378 males were checked for rubella IgG antibody using enzyme linked immunosorbent assay (ELISA) technique. $58.1 \%$ of the 770 cases were seropositive. $100 \%$ of the newborns were seropositive because of the maternal-derived antibodies. This trend decreased with time and finally reached zero by the age of 9 months. Then from the age of $4 y$, it started to increase and in the 15-19 years group peaked at $93 \%$.After that the seropositivity decreased to $85.7 \%$ in the age group of $>35$ years (contrary to our result). $89.6 \%$ of the newborns were seropositive. This figure (89.6\%) was less than our result (94\%). No statistically significant difference in seroprevalence was found between the two sexes. ${ }^{6}$

A descriptive study 7 was carried out to test the level of rubella immunity among 420 randomly selected women of reproductive age Serum anti-rubella antibody (IgG) was checked using ELISA method. The results showed that immunity rate ranged from $89.94 \%$ to $97.43 \%$ (an average of $94.05 \%)^{7}{ }^{7}$ This figure is in agreement with our result.

In order to assess the prevalence of immunity to rubella in pregnant women of Ahwaz city, specific antibody against rubella was determined using ELISA method in 250 serum samples of different age groups of pregnant women residing in Ahwaz. The result showed that $8 \%$ and $92 \%$ of these women were sensitive and immune to rubella respectively. 8 This figure (92\%) is less than our result (94\%).
$92.8 \%$ and $90.7 \%$ of women were immune in households and employers, respectively. ${ }^{8}$ There was no significant difference between immune households and employers. This agrees with our result. Contrary to our result, a study in Kerman 9 showed a statistically significant association between immune households and employers.

A study in Arak 10 showed $80.2 \%$ of women were immune against rubella and $19.8 \%$ were sensitive for rubella. This figure $(80.2 \%)$ was less than our result (94\%). Contrary to our result, a positive significant relationship between age and immunity was observed $(\mathrm{P}=0.00058) .{ }^{10}$ But there was not a significant relationship between place of living and immunity to rubella. 10This is in agreement with our result. A study was carried out to assess seroprevalence of rubella in primary school children of Tehran. 11 Five hundred and twenty-five subjects (31.5\%) out of 1665 were immunized against rubella, and 1140 subjects $(58.5 \%)$ had no antibody titer against rubella. Five hundred and fortyone $(32.5 \%)$ cases had a history of rubella immunization. Among vaccinated children, 205 subjects $(38 \%)$ were seropositive, while in non-vaccinated children 328 subjects $(29.2 \%)$ were seropositive.

They concluded vaccination caused no long term immunity. So, rubella immunization in infancy does not reduce congenital rubella. It seems that the best approach to prevent congenital rubella syndrome is to immunize girls shortly before marriage. ${ }^{11}$

A review of the epidemiology articles of clinical rubella in the Perm region of the Russian Federation 12 found that the incidence was about 220 cases per 100,000 populations. Rubella malformation was responsible for $15 \%$ of birth anomalies and about 3.5 cases of CRS per 1000 live births per year. Assessment of the seroepidemiology of rubella infection showed that the susceptibility rate among pregnant women was $16.5 \%$. This figure $(16.5 \%)$ was more than our results $(6 \%)$. Semerikov et al. ${ }^{12}$ found that pre- existing rubella antibodies do not interfere with the immune response to immunization, therefore selective immunization was provided to girls approaching puberty and to women of childbearing age. ${ }^{12}$

Information on the seroepidemiology of rubella in tropical African countries is still insufficient. A 6 year retrospective study was conducted to assess the seroprevalence in Senegal among women of child bearing age in the urban region of Dakar, Senegal. 13 The total seroprevalence calculated with a commercial enzyme immunoassay among 3471 serological results was $90.1 \%$. This figure $(90.1 \%)$ was less than our results (94\%). There was no significant difference between the years of study, age groups, and the socio-economic level of the patients. Compared to seroepidemiological studies conducted in other western African countries, their data suggest an important and stable circulation of the virus in the region of Dakar. The lack of information on rubella and congenital rubella syndrome (CRS) in Senegal must encourage medical authorities to establish a national rubella surveillance team to implement policies for the development of programs for the control and prevention of CRS in the country. ${ }^{13}$

On the other hand, most of the countries in Western Europe have now performed mass infant rubella immunization programs instead of or in addition to selective immunization to limited the risk of congenital rubella syndrome. The European countries Denmark, England and Wales, Finland, France, Germany, Italy and the Netherlands undertook large, national serological studies collecting several thousand serum specimens during 1994-1998. ${ }^{14}$ Several enzyme immuno-assays detected antibodies against rubella virus. Comparability of the assay results was obtained by standardized methodology. The results showed widely differing levels of immunity to rubella both in the general population and in the specific age groups of males and females. A low 
rate $(<5 \%)$ of susceptible in childhood and adolescents of both sexes was reported only in Finland and the Netherlands. In countries such as Italy with only moderate coverage for the infant vaccination program. There is now both high susceptibility levels in the general population and in the at-risk population. Likelihood of continued epidemics of rubella associated with cases of congenital rubella syndrome also exists. The continued program of selective immunization will help to deviate the effect of this transmission and protect women on reaching childbearing age. ${ }^{14}$

Our results showed that by the time women in Birjand reach childbearing age, $94 \%$ are immune to rubella. In another study found the risk of fetal infection was nearly $8 \%$ following re-infection in the first 16 weeks of pregnancy, but fetal malformations were scarce. ${ }^{15}$

Rubella immunization has not been advocated for inclusion in the Expanded Programmers for Immunization (EPI) in many developing countries. This is because where sustained high coverage cannot be guaranteed; its introduction could cause an unexpected increase in the number of susceptible young women by slowing but not interrupting virus transmission and thus shifting the age of first exposure into the reproductive years. ${ }^{16}$

Recently Shih et al. ${ }^{1}$ showed women who received one dose rubella immunization at preschool and 15-months-old have the highest seronegativities. They advocated a revised immunization strategy to women who received one dose rubella immunization at a younger age. ${ }^{1}$

In another study in the northern Mexican, city of Durango, the rate of rubella immunity in pregnant women was shown to be high. However, nearly $3 \%$ of pregnant women were susceptible to rubella in their setting. Risk factors associated with rubella seropositivity found in this study may be useful for optimal design of preventive measures against rubella and CRS. ${ }^{17}$

Ngaovithunvong et al. ${ }^{18} \mathrm{~s}$ findings correspond well with the immunization schedules, as the highest seropositivity rate was found in children between 0 and 7 years of age. For those older than 7 , there was a decrease in seropositivity rate despite good immunization coverage, and reaching its lowest rate in the 15-19-year age group. This showed that certain population groups might be incompletely vaccinated, or the humoral immunity produced by immunization gradually decreased over time. They advocated a booster dose of MMR vaccine for Thai adolescents in order to prevent future mumps epidemics.

Jablonka et al. ${ }^{19}$ suggested overall satisfactory MMRV immunity in adult immigrants coming to Europe, however, MMRV seroprevalence was low in refugee children, which supports the need for thorough and prompt immunization of young migrants entering Europe. Overall, their information set underlines the urgent need for implementation and validation of a immunization strategies for asylum seeker care in the current crisis. ${ }^{19}$

Since 2014 the number of refugees, arriving in Germany has increased rapidly and cases of vaccine-preventable diseases have been reported at reception centres 20 . In one study, refugees, 12 years and older arriving in Lower Saxony were serologically screened for antibodies against measles, rubella and varicella between November 2014 and October 2015. 20 Seroprevalence was determined from the screening data by disease, country of origin and age group and then compared to literature-based herd immunity thresholds to determine vaccination gaps. In total, 23,647 specimens were included in this study. Though the vast majority of refugees screened positive for antibodies against measles, rubella and varicella, the seroprevalences were not sufficient to ensure herd immunity 20 . The seroprevalence varied between countries of origin and increased with age. Refugees need to be vaccinated against measles, rubella and varicella and complete details of seroprevalence among subgroups of refugees and their immunizations must be provided at reception centers. ${ }^{20}$

Immunization finally is the best method of eliminating CRS. Two immunization master plans can be carried out.

A. A selective immunization allowing rubella to continue circulating.

B. Public immunization of children which has the goal of preventing both rubella and congenital rubella syndrome and has been found to be the more successful Tactic. ${ }^{2}$

\section{Conclusion}

This study is a stride in assessing the seroprevalence of rubella infection in women of childbearing age in Birjand, Iran. Although the present study showed that the level of immunity among women in Birjand is greater than the predicated critical level, but, it is advocated that all the women be checked serologically for $\operatorname{IgG}$ against rubella virus prior to marriage or pregnancy and seronegative ones be vaccinated against rubella.

"To be, or not to be, that is the question:

Whether "tis nobler in the mind to suffer....." is the opening phrase of a soliloquy in the "Nunnery Scene" of "Great Wiseman William Shakespeare's play Hamlet"

Finale, we also say: Rubella: To be or not to be, that is the question...

\section{Acknowledgments}

I wish to thank Dr. Hosseini without whose help this study could not have been completed. We would also to thank all participating patients for their cooperations and for their permission to publish this article.

\section{Conflicts of interest}

The authors declare there is no conflict of interests.

\section{Funding}

None.

\section{References}

1. Shih CT, Chang YC, Wang HL, et al. Comparing the rubella seronegativity in pregnant women who received one dose of rubella vaccine at different ages in Taiwan. Vaccine. 2016;34(40):4787-4791.

2. Corcoran C, Hardie DR. Seroprevalence of rubella antibodies among antenatal patients in the Western Cape. S Afr Med J. 2005;95(9):688690 .

3. Ki M, Youl Choi B, Kim M-H, et al. Rubella seroprevalence in Korean Children. J Korean Med Sci. 2003;18(3):331-336.

4. Cutts FT, Vynnycky E. Modelling the incidence of congenital rubella syndrome in developing countries. Int J Epidemiol. 1999;28(6):11761184.

5. Tabatabaei H, Vazirian P, Sarijoloo M, et al. Role of Rubella in patients with acute febrile rash in different parts of Iran. Hakim Research Journal. 2002;3(5):189-194. 
6. Eftekhar Ardebilli H, Salarilak SH, Farokh Eslamlou HR, et al. Seroepidemical survey on Rubella and characterizing the best strategy for immunization. Journal of School of Public Health And Institute Of Public Health Researches. 2002;1(1):1-8.

7. Ziaee S. A study on the level of immunity against Rubella virus among reproductive aged women in Kazeroon, 2000-2001. Scientific Medical Journal of Ahwaz University of Medical Sciences. 2003;39:1-6.

8. Ghafourian Boroujerdnia M, Mohammad Ghafari R, Khodadady A. Seroepidemiology of Rubella among pregnant women in Ahwaz, Iran. Scientific Medical Journal of Ahwaz University of Medical Sciences. 2003;38:1-8

9. Ashraf Ganjoei T, Mohammadi MM. Determination of Rubella virus antibodies in pregnant women referring to Kerman Maternity Hospitals in 1999. Medical Journal of Reproduction \& Infertility. 2001;6(2):2228.

10. Sufian M. Determination of immunity for Rubella among females in the year of marriage done in Arak (2000). Journal of Arak University of Medical Sciences. 2002;19(5):16-19.

11. Zamani A, Daneshjo KH. The Sero-epidemiological survey of rubella in primary school children of Tehran. Iranian Journal of Pediatrics. 2014;14(1):33-39.

12. Semerikov VV, Lavrentyeva IN, Popov VF, et al. Rubella in the Russian Federation:epidemiological features and control measures to prevent the congenital rubella syndrome. Epidemiol Infect. 2000;125(2):359-366.
13. Dromigny JA, Nabeth P, Perrier Gros Claude JD. Evaluation of the seroprevalence of rubella in the region of Dakar (Senegal). Trop Med Int Health. 2013;8(8):740-743.

14. Pebody RG, Edmunds WJ, Conyn-van Spaendonck M, et al. The seroepidemiology of rubella in western Europe. Epidemiol Infect. 2001;125(2):347-357.

15. Best JM, Banatvala JE. Rubella. In: Zuckerman AJ, editor. Principles and Practice of Clinical Virology. Chichester: John Wiley, USA; 2009:387-418.

16. Robertson SE, Cutts FT, Samuel R, et al. Vaccination against rubella. Bull World Health Organ. 1997;75(1):69-81.

17. Alvarado-Esquivel C, Hernandez-Tinoco J, Sanchez-Anguiano LF, et al. Rubella Immune Status in Pregnant Women in a Northern Mexican City. J Clin Med Res. 2016;8(9):656-661.

18. Ngaovithunvong V, Wanlapakorn N, Tesapirat L, et al. Mumps antibody in the Thai population 17 years after the universal measles mumps rubella vaccination program. J Infect Dev Ctries. 2016;10(7):735-740.

19. Jablonka A, Happle C, Grote U, et al. Measles, mumps, rubella, and varicella seroprevalence in refugees in Germany in 2015. Infection. 2016.

20. Toikkanen SE, Baillot A, Dreesman J, et al. Seroprevalence of Antibodies against Measles, Rubella and Varicella among Asylum Seekers Arriving in Lower Saxony, Germany, November 2014-October 2015. Int J Environ Res Public Health. 2016;13(7):E650. 